$\overline{\mathrm{AEET}}$

ASOCIACIÓN ESPAÑOLA DE ECOLOGÍA TERRESTRE

\title{
¿Estamos cumpliendo los objetivos que nos marcamos?
}

\author{
L. Cayuela ${ }^{1, *}$, Á. Alonso ${ }^{3}$, J.A. Blanco ${ }^{4}$, C. Puerta-Piñero ${ }^{5}$, S. Rodríguez-Echeverría ${ }^{6}$, L. Jiménez-Eguizabal ${ }^{2}$
}

(1) Departamento de Biología y Geología, ESCET, Universidad Rey Juan Carlos, c/ Tulipán s/n, 28933 Móstoles, Madrid, España

(2) Asociación Española de Ecología Terrestre (AEET), Departamento de Biología y Geología, Universidad Rey Juan Carlos, c/ Tulipán s/n, 28933 Móstoles, Madrid, España.

(3) Departamento de Ciencias de la Vida, Unidad Docente de Ecología, Universidad de Alcalá, Crta. N-II Km 33,6 Campus Universitario, Edificio Biología, 28871 Alcalá de Henares, Madrid, España.

(4) Departamento de Ciencias del Medio Natural, ETSIA, Universidad Pública de Navarra, Campus de Arrosadía s/n, 31006 , Pamplona, Navarra, España. (5) Instituto Andaluz de Investigación y Formación Agraria, Pesquera, Alimentaria y de la Producción Ecológica (IFAPA). Camino de Purchil s/n, 18004, Granada, España.

(6) Centro de Ecología Funcional. Departamento de Ciências da Vida. Universidad de Coimbra. 3000-455. Coimbra. Portugal.

* Autor de correpondencia: L. Cayuela [luis.cayuela@urjc.es]

Cayuela, L., Alonso, Á., Blanco, J.A., Puerta-Piñero, C., Rodríguez-Echeverría., Martín, N., Jiménez-Eguizábal, L. 2014. ¿Estamos cumpliendo los objetivos que nos marcamos?. Ecosistemas 23(1):61-63. Doi.: 10.7818/ECOS.2014.23-1.09

La gestión de una revista científica tiene que ser eficiente y lo suficientemente flexible como para adaptarse a los cambios. Para ello, es necesario que los objetivos marcados por el comité editorial sean claros y contrastables, y su grado de cumplimiento pueda ser cuantificado a lo largo del tiempo. En diciembre de 2012, en la primera editorial del actual comité de la revista, se planteaba una serie de objetivos que involucraban varios cambios en contenido y forma, y que buscaban en última instancia continuar mejorando la calidad de Ecosistemas (Cayuela et al. 2012a). En esta editorial se quiere hacer un balance del grado de cumplimiento de estos objetivos, tras casi año y medio de andadura.

En aquella primera editorial se explicaba la evolución que, de forma no consciente, la revista había sufrido en los últimos años, pasando de ser una revista exclusivamente divulgativa en sus inicios, allá por el año 1992, a una revista con todas las componentes propias de las revistas de investigación (Cayuela et al. 2012b). Con aquella primera editorial queríamos que el mensaje de que Ecosistemas fuera considerada como una revista de investigación calara entre nuestro público. Para evaluar el grado de cumplimiento de este objetivo, lo primero que hemos mirado ha sido el número de descargas de aquellas dos editoriales que explicaban la nueva política editorial de Ecosistemas (Cayuela et al. 2012b) y los objetivos que nos proponíamos cumplir en esta nueva etapa de la revista (Cayuela et al. 2012a). Es razonable asumir que si nadie ha leído las editoriales, el mensaje no llegue más allá de nuestra capacidad para difundirlo con la palabra, y se creen falsas expectativas tanto para los lectores de la revista como para los autores que envían sus contribuciones a la misma. En este periodo se realizaron 643 y 623 descargas para las dos editoriales arriba mencionadas, respectivamente, un número nada desdeñable que se puede poner mejor en contexto cuando se compara con las algo menos de 2800 descargas que ha tenido el artículo más visto durante los dos últimos años. Se decidió también hacer una encuesta a los lectores de la revista, que se difundió por la lista de distribución de usuarios de la revista, la lista de distribución de la Asociación Española de Ecología Terrestre (AEET), así como por las redes sociales (facebook, twitter). A esta encuesta contestaron 100 personas, de las cuales un $75.0 \%$ respondieron que la nueva línea editorial de la revista les gusta más que antes, un $24.0 \%$ respondieron que no ven grandes cambios con respecto a años anteriores, y tan sólo un $1.0 \%$ que les gusta menos que antes (Fig. 1a). Sin embargo el perfil científico que buscamos no ha quedado suficientemente claro entre nuestros lectores, ya que en contraste con el $82.8 \%$ de los lectores que buscan contenidos científicos en la revista, el $64.6 \%$ también esperan encontrar contenidos divulgativos, y un $18.2 \%$ contenidos técnicos (Fig. 1b). Es posible, no obstante, que la búsqueda de contenidos divulgativos responda al alto porcentaje de artículos de revisión que se publican en los monográficos, lo que es considerado por mucha gente como "alta divulgación". Con todo, parece que el perfil científico de la revista se va consolidando poco a poco, lo que sin duda son buenas noticias y nos anima a seguir con nuestro empeño.

Una de las grandes novedades introducidas por este comité editorial fue la transición a un sistema electrónico para la gestión de los artículos. Argumentábamos en su día que esto nos permitiría llevar a cabo una gestión más eficiente de los artículos. Nos inclinamos por el Open Journal System (OJS, http://pkp.sfu.ca/ojs/), que es un software de código abierto para la administración de revistas creado por el Public Knowledge Project (http://pkp.sfu.ca/). A finales del 2012 hicimos la migración de los contenidos de la revista a este sistema. Reconocemos que los comienzos han sido duros para todos, y en especial para los autores, revisores y editores invitados, debido a que la aplicación ofrecía demasiadas posibilidades y no siempre muy obvias. Durante todo este tiempo hemos ido recibiendo críticas y comentarios constructivos de los usuarios y hemos ido modificando la aplicación para hacerla más intuitiva y fácil de usar. Como resultado de este proceso, se han mejorado mucho las interfaces para los autores, revisores y editores con respecto a las que implementaba el propio sistema por defecto. También hemos ganado en agilidad a la hora de gestionar los manuscritos como editores. Prueba de ello, es que el tiempo medio de revisión desde que se recibe un artículo hasta que se revisa es ahora de 40 días, labor que hubiera sido impensable sin el inconmensurable esfuerzo de los revisores, a los que agradecemos enormemente su labor y profesionalidad. Otra novedad que hemos incluido durante este último año es la presencia de un apartado en 
la página principal de la revista con los artículos más descargados durante los dos últimos años. Sin duda esperamos seguir mejorando la funcionalidad del sistema y continuar recibiendo las críticas y comentarios de nuestros usuarios, que como siempre, son las que nos hacen avanzar hacia la excelencia.

La implementación del DOI (Digital Object Identifier) también ha aumentado la accesibilidad de los artículos publicados en la revista. Gracias al DOI buscadores como Google Scholar encuentran ahora de forma inmediata los artículos publicados en Ecosistemas. Se hace difícil, sin embargo, discernir cual es la contribución de este cambio a la visibilidad de la revista, medida en términos de número de visitas o descargas. En 2013 la revista tuvo un total de 79961 visitas, la mayoría de España $(24.5 \%)$ y países lationamericanos como México (28.3\%), Colombia (12.9\%), Argentina (6.2 \%) o Perú $(6.0 \%)$. Se mantiene, como ya habíamos apuntado en otra editorial (Cayuela et al. 2013), nuestra hegemonía como revista científica de referencia en ecología en Latinoamérica, lo que refuerza nuestra posición de mantener la publicación de artículos escritos exclusivamente en castellano.

A este último respecto, seguimos recibiendo sugerencias para que Ecosistemas se abra a la publicación de artículos tanto en castellano como en inglés, como hacen algunas revistas científicas. Aunque es una posibilidad a la que no cerramos la puerta en el futuro, en el presente y dado el público objetivo que tenemos, creemos que la publicación en castellano es una marca distintiva que nos posiciona entre nuestros lectores, en particular en el ámbito latinoamericano. No obstante, se ha decidido que a partir de este número los pies de tablas y figuras irán en ambos idiomas. Esto, sumado a la publicación del resumen y palabras clave en castellano e inglés, ofrece un mayor grado de internacionalización a la revista, especialmente entre el público no hispanohablante, y por tanto esperamos que su visibilidad e impacto en la comunidad científica aumente.

Uno de los grandes retos que nos propusimos fue obtener la indexación de Ecosistemas en Science Citation Index (SCI) publicado por ThomsonReuters. Pues bien, la revista ya se encuentra en proceso de evaluación. Este proceso durará entre uno y dos años. Si tuviéramos factor de impacto, este sería en la actualidad de 0.22 , calculado con base en las citas recibidas durante 2013 para artículos publicados durante los años 2011 y 2012 . Es un factor de impacto pequeño que podría aumentar si los artículos que publicamos son cada vez de mayor relevancia científica. Además, si somos capaces entre todos de romper con ese aparente complejo de citar en los trabajos científicos internacionales artículos publicados en lengua castellana, el posicionamiento de nuestra revista mejoraría. Por suerte, y aunque con paso lento, se va avanzando en este sentido. En la encuesta realizada, solo un $1 \%$ de los encuestados contestaron que citaban los artículos publicados en Ecosistemas con bastante frecuencia (4 o más veces cada 10 trabajos publicados), un $17.3 \%$ eventualmente ( $2-3$ veces cada 10 trabajos publicados), un $42.9 \%$ con poca frecuencia ( 1 vez cada 10 trabajos publicados) y un $31.6 \%$ nunca (Fig. 1c). Obviamente la causa de no citar más artículos de Ecosistemas podría responder a que no hay publicado nada relevante en la materia que se está investigando. $Y$ aunque no fuera así, los datos invitan a un cierto optimismo, ya que incluso en el peor de los escenarios (1 cita en promedio cada 10 artículos), la revista podría aumentar su impacto durante los próximos años de forma considerable. En cualquier caso, ya explicamos que nuestro interés en este sentido no es otro que el de captar el interés de autores para que envíen sus contribuciones a la revista. Esto es relevante en un mundo cada vez más mediatizado por los índices de impacto, pero sin duda coincidimos en que la calidad no la da necesariamente el factor de impacto (Hendee et al. 2012), y de que se puede sacar adelante de forma muy digna una revista científica de calidad sin necesidad de que esté en SCl.

Somos conscientes de que las críticas al factor de impacto de Thomson Reuters (o de su alternativa en el Scimago Journal Rank publicado por Elsevier) son muchas y variadas (Seglen 1997; Mistelli 2013). En resumen, la puntuación de la revista es una media de todos los trabajos publicados en la misma y dice poco de la ca- lidad de cualquier investigación concreta. Somos conscientes de estas limitaciones de los factores de impacto, pero también tenemos claro que a los profesionales de la ciencia se les suele juzgar la calidad y productividad de su trabajo con base en el número de publicaciones y al índice de impacto de las revistas donde publican. Creemos, por tanto, que obtener la indexación en $\mathrm{SCl}$ puede beneficiar a los autores de artículos publicados en Ecosistemas. Sin embargo, queremos dejar claro que el objetivo de nuestra revista no es obtener una puntuación concreta, sino convertirnos en un referente de buena ciencia ecológica escrita en castellano. Queremos hacernos eco de las palabras de Randy Schekman (Premio Nobel de Medicina 2013): "hay una vía mejor, gracias a la nueva remesa de revistas de libre acceso que son gratuitas para cualquiera que quiera leerlas y no tienen caras suscripciones que promover. Nacidas en Internet, pueden aceptar todos los artículos que cumplan unas normas de calidad, sin topes artificiales. Muchas están dirigidas por científicos en activo [o promovidas por sociedades científicas], capaces de calibrar el valor de los artículos sin tener en cuenta las citas" (El País 12-12-13). Creemos que esta es justamente la característica de Ecosistemas.

Conseguir la indexación contribuiría sin duda a garantizar los contenidos de la revista a largo plazo. En este sentido hemos sido bastante exitosos en el último año en la búsqueda de editores invitados que coordinen números monográficos, pero lamentablemente seguimos teniendo un número muy reducido de artículos fuera de los monográficos, y en particular artículos con la suficiente calidad científica como para que puedan ser publicados. Si conseguimos la indexación esperamos revertir esta tendencia, aunque los resultados de la prospección realizada entre los usuarios de la revista nos permite, una vez más, ser optimistas. Ante la pregunta de si consideraban la posibilidad de enviar alguno de sus trabajos de investigación y/o revisión a Ecosistemas, un $13 \%$ de los encuestados contestaron que era poco probable, un $10 \%$ que sólo si era por invitación para contribuir a un número monográfico, un $25 \%$ que sólo si la revista estuviera indexada en $\mathrm{SCl}$, y un $45 \%$ que sí, en cualquiera de los casos (Fig.1d). Esperamos que realmente esto sea así y poder recibir un número cada vez mayor de contribuciones de calidad. En lo que se refiere a los monográficos, tenemos ya programada la publicación de los números de 2014 y varias ideas en marcha para 2015. Agradecemos a los editores invitados su buen quehacer en esta tarea tan laboriosa. Es gracias a los números monográficos que la revista se llena de contenidos, y es posiblemente este carácter monotemático lo que la hace atractiva para muchos de nuestros lectores. Desde estas líneas animamos a científicos e investigadores que nos propongan ideas para futuros monográficos.

Ecosistemas se está terminando de consolidar, decíamos al principio, como una revista de investigación, y esto ha causado cierta preocupación en algunos de nuestros lectores, a los que les gustaba el carácter divulgativo de la misma. Es por ello, y por nuestra creencia de que la divulgación de la ciencia juega un papel tan importante como el desarrollo de la misma, que en paralelo a todos estos cambios iniciamos a finales de 2012 el blog de la Revista Ecosistemas (http://revistaecosistemasblog.net). Inicialmente contábamos con una Editora de Comunicación que se encargaba de redactar entradas en el blog relacionadas con los artículos publicados en la revista ¿Qué artículos? Aquellos que considerábamos podrían atraer a un público más amplio. Las entradas eran escritas en un lenguaje llano y asequible. Pronto a esta actividad se sumó, como parte de la estrategia de comunicación de la AEET, la empresa Meta Comunica, que en la actualidad coordina y participa en la labor divulgativa y de difusión que se realiza desde el blog. En la actualidad publicamos una entrada mensual, siempre en relación a los contenidos publicados en la revista, y podemos decir con bastante rotundidad que estamos siendo exitosos con la divulgación de los contenidos que publicamos en Ecosistemas, ya que el blog ha recibido desde su creación un total de 11753 visitas, lo que supone un promedio durante los últimos seis meses de cerca de 1000 visitas al mes. Además, se han redactado seis notas de prensa y 
a)

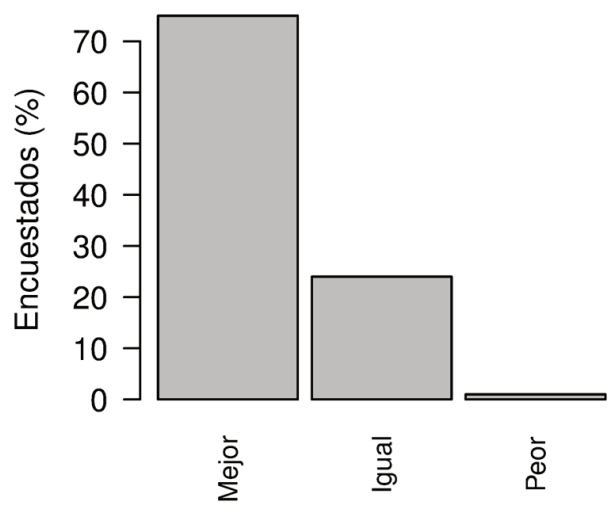

c)

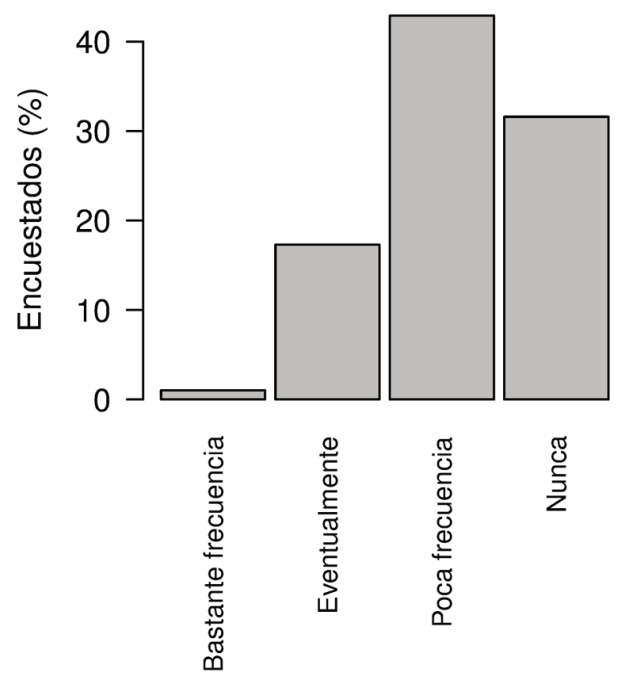

b)

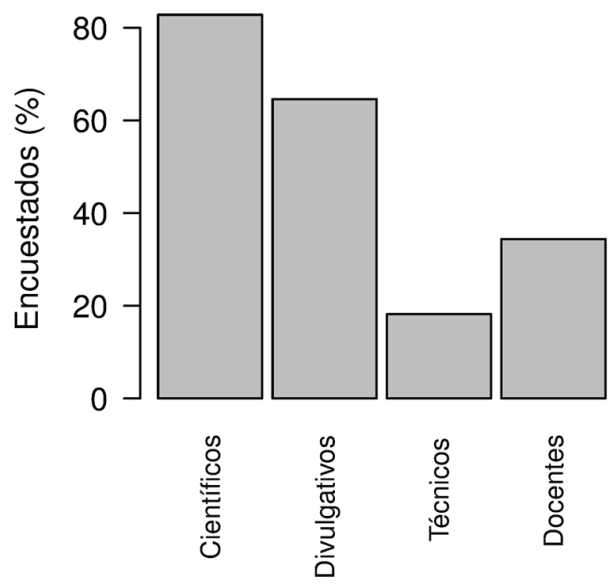

d)

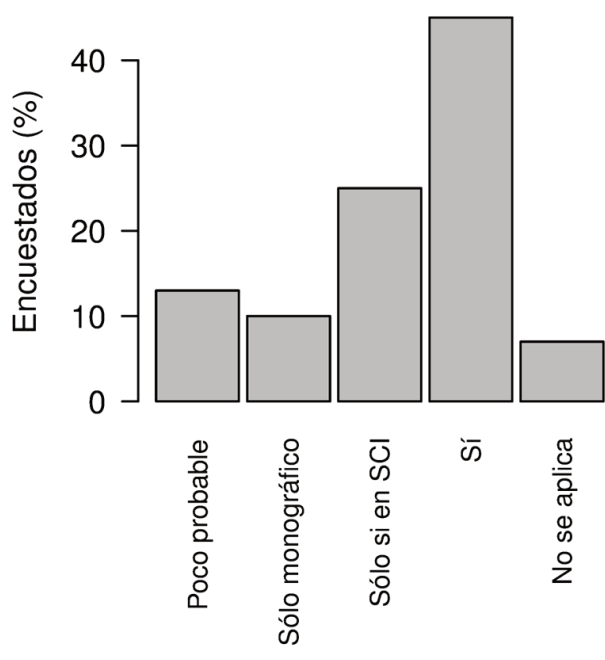

Figura 1. Porcentaje de encuestados que respondieron a la pregunta de (a) qué les parecía, con respecto a la anterior, la nueva línea editorial de Ecosistemas; (b) qué tipos de contenidos buscan en la revista; (c) con qué frecuencia citan los artículos publicados en Ecosistemas: (bastante frecuencia [4 o más veces cada 10 trabajos publicados], eventualmente [2-3 veces cada 10 trabajos publicados], poca frecuencia [1 vez cada 10 trabajos publicados], o nunca); (d) si consideran la posibilidad de enviar alguno de sus trabajos de investigación y/o revisión a Ecosistemas.

publicado alrededor de 60 artículos relacionados con investigaciones publicadas en nuestra revista en agencias como Efe Verde y Europa Press, en periódicos como El País, La Razón y La Vanguardia, así como entrevistas en cadenas de radio como Cadena Cope. Esto supone un modelo casi único en revistas científicas, ya que aquellas que lo tienen, dejan en manos de los propios autores la labor divulgativa, y nosotros creemos que la divulgación es más efectiva, en la mayoría de los casos, si se realiza por expertos en comunicación. Los autores que envíen sus contribuciones a Ecosistemas también deben considerar este aspecto como un valor añadido que magnifica el impacto social de sus artículos.

En resumen, creemos que hemos cumplido, o estamos en vías de cumplir, la mayoría de los objetivos que nos planteamos hace tan sólo un año y medio. Reconocemos que hay mucho trabajo por delante y afrontamos la siguiente etapa con entusiasmo. Aún tenemos muchas ideas y ganas de innovar y queremos que, de la mano de lectores, autores, revisores y editores invitados, sigamos construyendo una revista cada vez de mayor calidad.

\section{Referencias}

Cayuela, L., Alonso, Á., Blanco, J.A., Puerta-Piñero, C., Rodríguez-Echeverría, S., Martín, N., Jiménez-Eguizábal, L. 2012a. Novedades en Ecosistemas. Ecosistemas 21(3): 97-99.

Cayuela, L., Alonso, Á., Blanco, J.A., Puerta-Piñero, C., Rodríguez-Echeverría, S., Jiménez-Eguizábal, L. 2012b. La política editorial de Ecosistemas. Ecosistemas 21(3): 100-102.

Cayuela, L., Alonso, Á., Escudero, A., Quero, J.L. 2013. Premio "Ecosistemas" al mejor resumen de Tesis Doctoral publicado en esta revista en 2012. Ecosistemas 22(1): 70.

Hendee, W., Bernstein, M.A., Levine, D. 2012. Scientific journals and impact factors. Skeletal Radiology 41: 127-128.

Mistelli, T. 2013. Eliminating the impact of the Impact Factor. Journal of Cell Biology 201: 651-652.

Seglen, O.P. 1997. Why the impact factor of journals should not be used for evaluating research. British Medical Journal 314: 497. 